\title{
Large-area microfocal spectroscopic ellipsometry mapping of thickness and electronic properties of epitaxial graphene on Si- and C-face of 3C-SiC(111)
}

\author{
Vanya Darakchieva, A. Boosalis, A. A. Zakharov, T. Hofmann, M. Schubert, T. E. Tiwald, \\ Tihomir Iakimov, Remigijus Vasiliauskas and Rositsa Yakimova
}

\section{Linköping University Post Print}

\section{Tweet}

N.B.: When citing this work, cite the original article.

Original Publication:

Vanya Darakchieva, A. Boosalis, A. A. Zakharov, T. Hofmann, M. Schubert, T. E. Tiwald, Tihomir Iakimov, Remigijus Vasiliauskas and Rositsa Yakimova, Large-area microfocal spectroscopic ellipsometry mapping of thickness and electronic properties of epitaxial graphene on Si- and C-face of 3C-SiC(111), 2013, Applied Physics Letters, (102), 21, 213116.

http://dx.doi.org/10.1063/1.4808379

Copyright: American Institute of Physics (AIP) http://www.aip.org/

Postprint available at: Linköping University Electronic Press http://urn.kb.se/resolve?urn=urn:nbn:se:liu:diva-96134 


\title{
Large-area microfocal spectroscopic ellipsometry mapping of thickness and electronic properties of epitaxial graphene on $\mathrm{Si}$ - and $\mathrm{C}$-face of $3 \mathrm{C}$-SiC(111)
}

\author{
V. Darakchieva, ${ }^{1, a)}$ A. Boosalis, ${ }^{2}$ A. A. Zakharov ${ }^{3}$ T. Hofmann, ${ }^{2}$ M. Schubert, ${ }^{2}$ T. E. Tiwald,${ }^{4}$ \\ T. lakimov, ${ }^{1}$ R. Vasiliauskas, ${ }^{1}$ and R. Yakimova ${ }^{1}$ \\ ${ }^{1}$ Department of Physics, Chemistry and Biology, IFM, Linköping University, SE-581 83 Linköping, Sweden \\ ${ }^{2}$ Department of Engineering, University of Nebraska-Lincoln, Lincoln, 68588-0511 Nebraska, USA \\ ${ }^{3}$ Lund University, Maxlab, S-22100 Lund, Sweden \\ ${ }^{4}$ J. A. Woollam Co., Lincoln, Nebraska, 68508-2243 USA
}

(Received 15 April 2013; accepted 17 May 2013; published online 31 May 2013)

\begin{abstract}
Microfocal spectroscopic ellipsometry mapping of the electronic properties and thickness of epitaxial graphene grown by high-temperature sublimation on 3C-SiC (111) substrates is reported. Growth of one monolayer graphene is demonstrated on both Si- and C-polarity of the 3C-SiC substrates and it is shown that large area homogeneous single monolayer graphene can be achieved on the Si-face substrates. Correlations between the number of graphene monolayers on one hand and the main transition associated with an exciton enhanced van Hove singularity at $\sim 4.5 \mathrm{eV}$ and the free-charge carrier scattering time, on the other are established. It is shown that the interface structure on the $\mathrm{Si}$ - and $\mathrm{C}$-polarity of the $3 \mathrm{C}$ - $\mathrm{SiC}(111)$ differs and has a determining role for the thickness and electronic properties homogeneity of the epitaxial graphene. (C) 2013 AIP Publishing LLC. [http://dx.doi.org/10.1063/1.4808379]
\end{abstract}

Epitaxial graphene (EG) grown by sublimation epitaxy on silicon carbide $(\mathrm{SiC})$ holds great promise for large-scale production of next generation fast electronic devices. ${ }^{1-7}$ Despite significant progress and intense research efforts in the field, state-of-the-art EG shows electronic mobility parameters that are still orders of magnitude lower than those found in exfoliated graphene. Understanding the physical origin of the substantially different transport properties of EG and exfoliated graphene remains one of the major issues, and prevents further technological advances. The key point is to identify and control how the substrate affects EG uniformity, thickness, layer stacking, and carrier mobility properties. Of particular interest is to monitor and control the thickness where EG can be composed of single layer graphene (SLG), few (FLG) or multiple (MLG) layer graphene. While EG on the hexagonal polytypes of $\mathrm{SiC}$ has been extensively studied, knowledge about growth and properties of EG on cubic $3 \mathrm{C}-\mathrm{SiC}$ substrates is not exhaustive. Very few studies on graphene growth on the Si-face of $3 \mathrm{C}-\mathrm{SiC}(111)$ on $\mathrm{Si}$ have been performed, and terrace growth of EG with domain sizes of few micrometers and limited homogeneity was reported. ${ }^{8,9}$ 3C-SiC offers a number of advantages over piezoelectric polytypes of $\mathrm{SiC}$ because of its isotropic and unpolarized growth surfaces and the reported performance improvement of SiC-based electronic devices.

The growth mechanisms and kinetics as well as the electronic and transport properties of EG grown on the two $(\mathrm{Si}$ and $\mathrm{C}$ ) polar faces of the hexagonal polytypes of $\mathrm{SiC}(0001)$ are significantly different. ${ }^{10,11}$ For example, on the Si-face of $4 \mathrm{H}-\mathrm{SiC}$ and $6 \mathrm{H}-\mathrm{SiC}$ a buffer layer is typically formed before the growth of graphene. The buffer layer which is strongly bonded to the substrate represents a $(6 \sqrt{3} \times 6 \sqrt{3}) \mathrm{R} 30^{\circ}$ surface reconstruction. On the other hand, the reconstruction on the $\mathrm{C}$-face may be different and the first layer is believed to

${ }^{\text {a)} E l e c t r o n i c ~ m a i l: ~ v a n y a @ i f m . l i u . s e ~}$ be weakly bonded to the substrate. Much larger domains with uniform EG thickness of SLG and sizes of several hundreds of micrometers are obtained on the $\mathrm{Si}$-face of $4 \mathrm{H}-\mathrm{SiC}$ and $6 \mathrm{H}-\mathrm{SiC} .{ }^{3}$ However, on a large scale, necessary for industrial applications, EG on Si-face still shows certain nonuniformity. EG on C-face shows smaller domains with sizes up to several tens of micrometers ${ }^{12}$ where the control of thickness down to SLG or FLG is difficult. The simultaneous mapping of these properties presents a significant challenge due to the varying domain structure of EG and the fact that characterization techniques with different spatial resolution are typically employed. In order to provide in-depth characterization of $\mathrm{EG}$ grown on $3 \mathrm{C}-\mathrm{SiC}$, we employ here a largescale mapping approach of structural and electronic properties. Our approach identifies functional correlations between structural, electronic and transport properties, and may be further used to reveal their interrelations with the influence of the substrate. Identifying these functional characteristics may ultimately enable device technologies.

Spectroscopic ellipsometry (SE) is a contactless, noninvasive technique well known for its high sensitivity to thin layers with sub-nm thickness. SE allows access to the dielectric function (DF) of individual constituents of layered heterostructures and it is widely employed in fundamental and applied materials research. ${ }^{13-16}$ The DF is governed by electronic transitions and provides the optical constants and information on the electronic structure of the material. SE in the near-infrared-visible-ultraviolet region was used to study EG obtained by different techniques, ${ }^{17-21}$ expfoliated graphene, ${ }^{22,23}$ as well as for in situ monitoring of graphene growth on metals. ${ }^{24}$ Imaging ellipsometry was used to determine thickness of small flakes of exfoliated graphene..$^{25-27}$ We have recently reported the visible-ultravacuum dielectric functions of EG grown on different polytypes of $\mathrm{SiC}$, where we developed a parameterized dielectric function model for graphene. ${ }^{21} \mathrm{SE}$ investigations at terahertz 
frequencies combined with optical Hall effect measurements revealed and quantified conductive channels in EG on $6 \mathrm{H}-\mathrm{SiC} .^{28}$ Large-area mapping of EG thickness and electronic properties using microfocal-SE $(\mu-\mathrm{SE})$ has not been reported yet.

In this work, we report on large-area $\mu$-SE mapping of thickness and electronic properties derived from the MDF of EG grown on thick bulk-like 3C-SiC(111) layers. Complementary low-energy electron microscopy (LEEM) and micro-low-energy electron diffraction ( $\mu$-LEED) measurements probe morphology, thickness, and the surface structure of the EG in selected sample locations. We explore the growth on $\mathrm{Si}$-face and $\mathrm{C}$-face of the $3 \mathrm{C}$-SiC substrate, and discuss the determining factors of EG thickness uniformity in relation to its structural, electronic, and transport properties.

EG samples were grown by high temperature sublimation in $\mathrm{Ar}_{2}$ atmosphere under optimized conditions on the Si- and C-face of 3C-SiC(111). The thick (few hundreds of micrometers) $3 \mathrm{C}-\mathrm{SiC}$ layers were grown by sublimation epitaxy on $6 \mathrm{H}-\mathrm{SiC}(0001)$ with our in-house-built reactor. ${ }^{29}$ $\mu$-SE mapping of circular areas with diameter of $0.5 \mathrm{~cm}$ for photon energies from $1.25 \mathrm{eV}$ to $5.45 \mathrm{eV}$ was performed with a multi-channel ellipsometer equipped with focusing optics allowing for measurement spot size of approximately $25 \times 50 \mu \mathrm{m}^{2}$ (M2000; J. A. Woollam Co., Inc.). LEEM and $\mu$-LEED measurements were performed on selected locations on the samples with resolution of $10 \mathrm{~nm}$. The number of layers can be extracted from the number of minima in the electron reflectivity spectra extracted from energy series of LEEM images. ${ }^{30}$ The LEEM image contrast taken at a given electron energy reveals information on the number of layers and domain structure. The field of view used for LEEM $(50 \mu \mathrm{m})$ matches approximately with the $\mu$-SE spot size and results obtained by LEEM and $\mu$-SE allow for comparative conclusions.

SE determines the ratio of the complex reflection coefficients $R_{p}$ and $R_{s}$ for light polarized parallel $(p)$ and perpendicular $(s)$ to the plane of incidence, respectively ${ }^{13}$

$$
\rho=R_{p} / R_{s}=\tan \Psi \exp (i \Delta),
$$

where $\Psi$ and $\Delta$ denote the ellipsometric angles. A regression analysis employing layer model calculations and model DF (MDF) approaches is necessary to obtain the EG thickness and MDF parameters. A stratified layer model (substrate/layer/ layer/ambient) composed of substrate, interface layer between the substrate and graphene, and graphene layer is used here. In our optical model, the interface layer accounts for a buffer layer (if present), roughness of the substrate surface (an effect of the slight off-axis cut of the $\mathrm{SiC}$ substrate), and non-uniform sublimation of silicon from the $\mathrm{SiC}$ substrate. All layers are treated isotropically in our model since ellipsometry has no sensitivity to the out-of-plane polarizability of ultra-thin layers. The MDFs of the 3C-SiC(111) substrate, as determined in our previous work, ${ }^{21}$ were implemented with no further changes. The graphene MDF, $\varepsilon_{E G}$ comprises electronic, $\varepsilon_{E}$, and freecharge carrier, $\varepsilon_{F C}$, contributions

$$
\varepsilon_{E G}=\varepsilon_{E}+\varepsilon_{F C} .
$$

$\varepsilon_{E}$ is composed of Lorentzian and Gaussian oscillators to describe the critical point $(\mathrm{CP})$ in the dielectric function associated with an exciton-enhanced van Hove singularity in the density of states at $\sim 4.5 \mathrm{eV} .^{21}$ The free-charge carrier contribution is described by a classical Drude model

$$
\varepsilon_{F C}=\varepsilon_{\infty}-\frac{i 4 \pi \hbar^{2}}{\rho\left(\hbar \lambda+i \lambda^{2} \tau\right)},
$$

where $\rho, \lambda$, and $\tau$ are the resistivity, the wavelength and the free-charge carrier scattering time, respectively. The freecharge carrier scattering time is related to the optical mobility parameter

$$
\tau=\frac{m_{e f f} \mu}{q},
$$

where $m_{e f f}, \mu$, and $q$ are the free-charge carrier effective mass, mobility and charge, respectively.

The interface layer MDF was treated as an effective medium approximation (EMA) between the MDFs of the substrate and graphene. The thickness of the interface layer was set to $0.35 \mathrm{~nm}$ (the thickness of SLG) without further variation during the analysis, but its constituents percentage was allowed to vary between $0 \%$ and $100 \%$. EMA of $100 \%$ stands for interface layer consisting entirely of SiC substrate and thus implying a smooth interface. In contrast, an intermediate EMA of $50 \%$ graphene and $50 \% \mathrm{SiC}$ would indicate an interface layer that is randomly rough. The parameters of the graphene MDF (Lorentzian and Gaussian oscillator amplitudes, energies and broadenings, free-charge-carrier scattering time and resistivity), the graphene layer thickness, and the interface-layer $\mathrm{SiC}$ percentage were varied until the experimental and calculated data matched. Excellent agreement between experimental and best-match calculated $\Psi$ and $\Delta$ spectra is achieved in all points of the maps with typical standard-deviation-weighted mean squared error below 0.1 .

Figure 1 shows best-match model calculated maps of graphene layer thickness, interface-layer $\mathrm{SiC}$ percentage, and free-charge-carrier scattering time parameters obtained from $\mu$-SE data from two EG samples grown on Si-face and C-face 3C-SiC(111). The thickness maps in Fig. 1(a) reveal large homogeneous areas $\sim 2 \times 2 \mathrm{~mm}^{2}$ with SLG on the Siface. In addition, few islands of MLG of several hundred micrometer size occur where the carbon bunched up on the surface of the substrate. Fig. 1(b) depicts a uniform and large interface-layer $\mathrm{SiC}$ percentage within the same regions where SLG was found on Si-face, which is indicative for a very smooth substrate surface. However, beneath the thick islands the interface layer was found to consist of approximately $\sim 50 \% \mathrm{SiC}$ indicative for significant surface roughness. In principle, this can be related to defects in the substrate surface. Indeed, we have identified surface depressions at the $\mathrm{Si}$-face of the $3 \mathrm{C}-\mathrm{SiC}$ substrate associated with twin boundaries. It is well known that defects in the $\mathrm{SiC}$ substrate serve as preferential centers for enhanced Si sublimation. ${ }^{31}$ Thus, a higher growth rate of graphene could be expected around these defects, which may explain the formation of such graphite-like islands. 

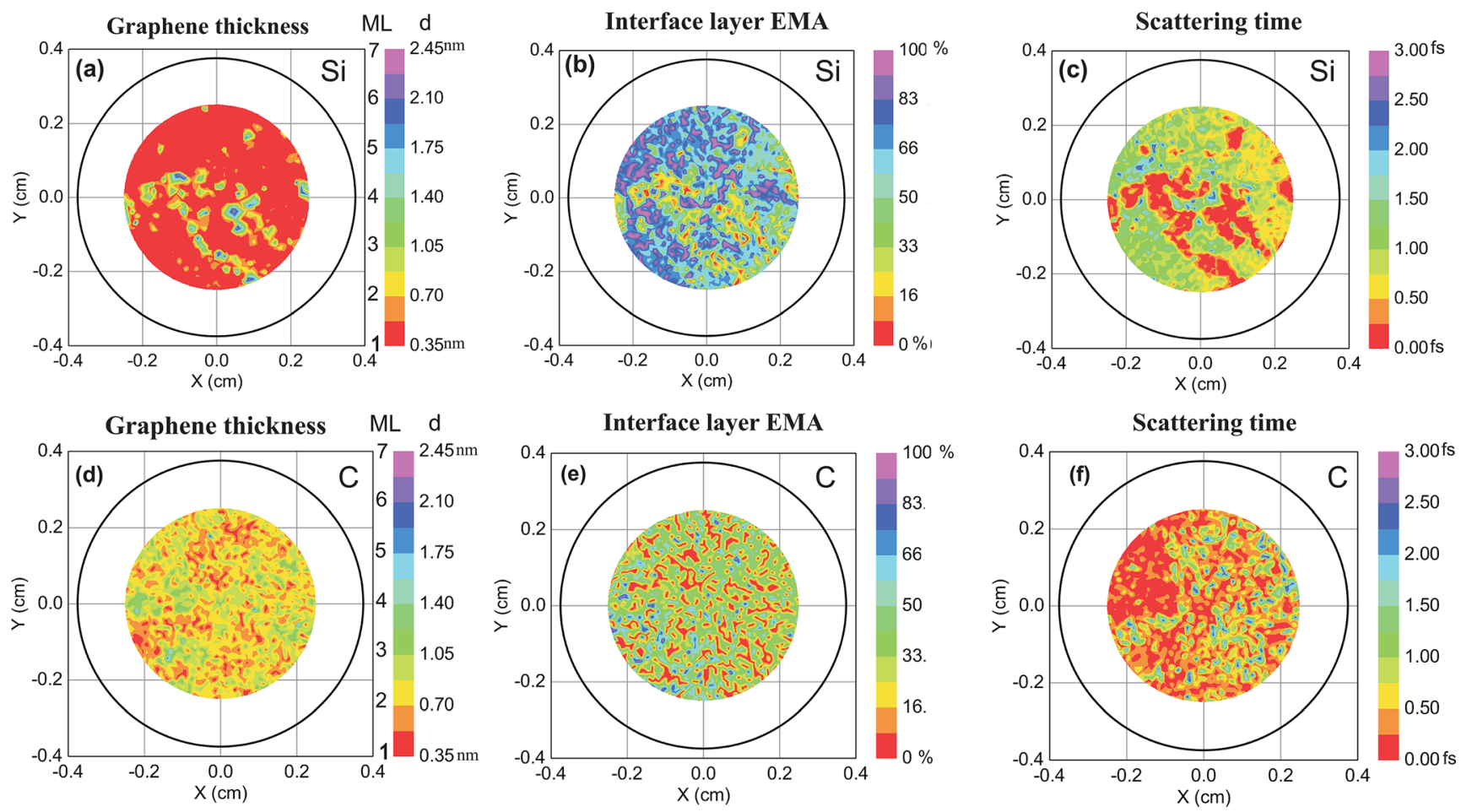

FIG. 1. $\mu$-SE maps of best-match model parameters for graphene layer thickness (a, d), interface-layer SiC percentage (b, e), and free-charge-carrier scattering time (c, f) for EG grown on Si-face (a, b, c) and C-face (d, e, f) of 3C-SiC.

The areas with homogeneous graphene layer thickness in Fig. 1(d) for C-face 3C-SiC(111) are much smaller, where the thickness varies mostly between 1 and 3 monolayer graphene, and the formation of large MLG islands is not detected. It has been shown that the growth of SLG on the C-face of the hexagonal $\mathrm{SiC}$ polytypes is very challenging due to the much higher sublimation rate of $\mathrm{Si}$ and usually growth of MLG is reported for these surfaces. Although SLG growth on C-face of $3 \mathrm{C}-\mathrm{SiC}(111)$ is achieved here, the domain size depicted in Fig. 1(d) remains rather small. Fig. 1(e) depicts an interfacelayer $\mathrm{SiC}$ percentage indicative for high substrate surface roughness and small uncorrelated islands of large graphene content. These results for $3 \mathrm{C}-\mathrm{SiC}(111)$ indicate that the interface structure of EG on $\mathrm{C}$-face differs distinctively from Si-face.

Fig. 2(a) shows an exemplary LEEM image for a region of the Si-face sample where a graphene thickness of approximately SLG was found from the $\mu$-SE maps. Large areas with a bright contrast occur, which can be associated with SLG, and a few small regions occur with a darker contrast and which can be related to two monolayers graphene. The LEEM image confirms the highly homogeneous areal growth of SLG in excellent agreement with the $\mu$-SE result. Fig. 2(c) shows LEED pattern taken from a SLG region, and reveals the $1 \times 1$ diffraction spots associated with SLG surrounded by the $6 \sqrt{3} \times 6 \sqrt{3}-\mathrm{R} 30^{\circ}$ diffraction spots associated with the $\mathrm{SiC}$ surface. These results suggest that a buffer layer similar to the one found on $\mathrm{Si}$-face $4 \mathrm{H}-\mathrm{SiC}$ and $6 \mathrm{H}-\mathrm{SiC}$ is formed on the smooth surface areas of the Si-face 3C-SiC(111).

Fig. 2(c) shows an exemplary LEEM image for a region of the C-face sample. The image shows domains of one to four monolayer graphene with dimensions below the resolution for the $\mu$-SE mapping. The much smaller domains of homogeneous graphene layers observed by LEEM [Fig. 1(d)] in this case may be related to the high substrate surface roughness found for C-face in Fig. 1(e). Fig. 1(d) presents LEED pattern taken from a SLG region on the C-face sample. Diffraction spots due to graphene occur only, and no superstructure related to a specific $\mathrm{SiC}$ surface reconstruction
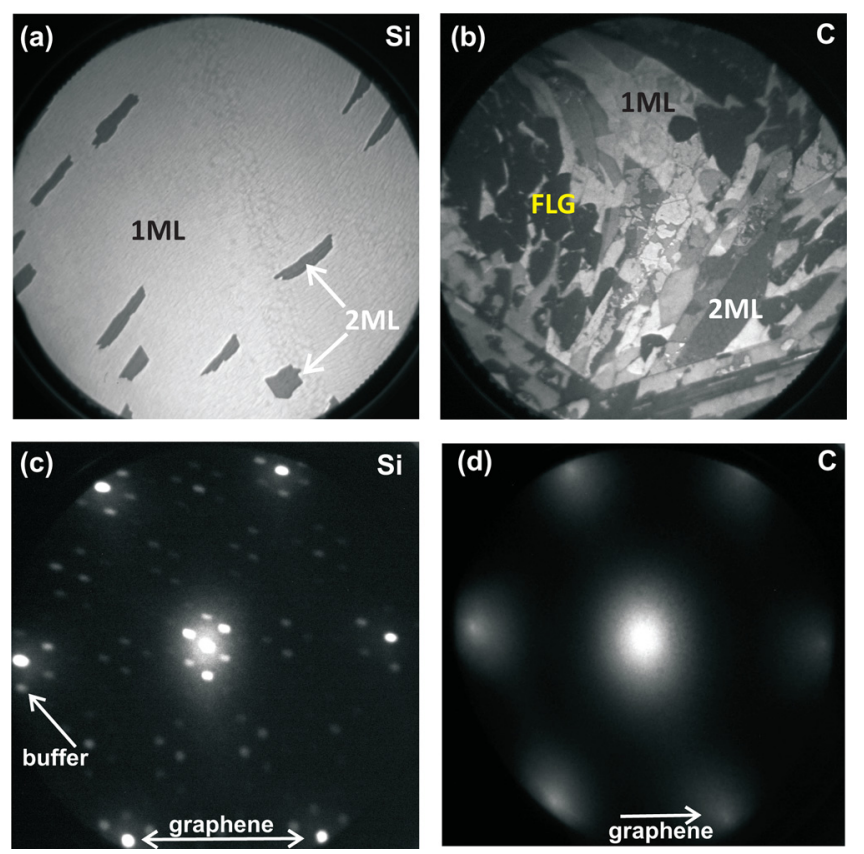

FIG. 2. LEEM images from selected sample areas for EG on Si-face (a) and C-face $3 \mathrm{C}-\mathrm{SiC}$ (b) (field of view $50 \mu \mathrm{m}$ ). Domains with 1, 2 and few monolayer (FML: 3 and 4 monolayers) graphene are indicated on the LEEM images. $\mu$-LEED pattern from a SLG (ML) area of the Si-face (c) and Cface (d) taken at 40 and $44 \mathrm{eV}$, respectively. 


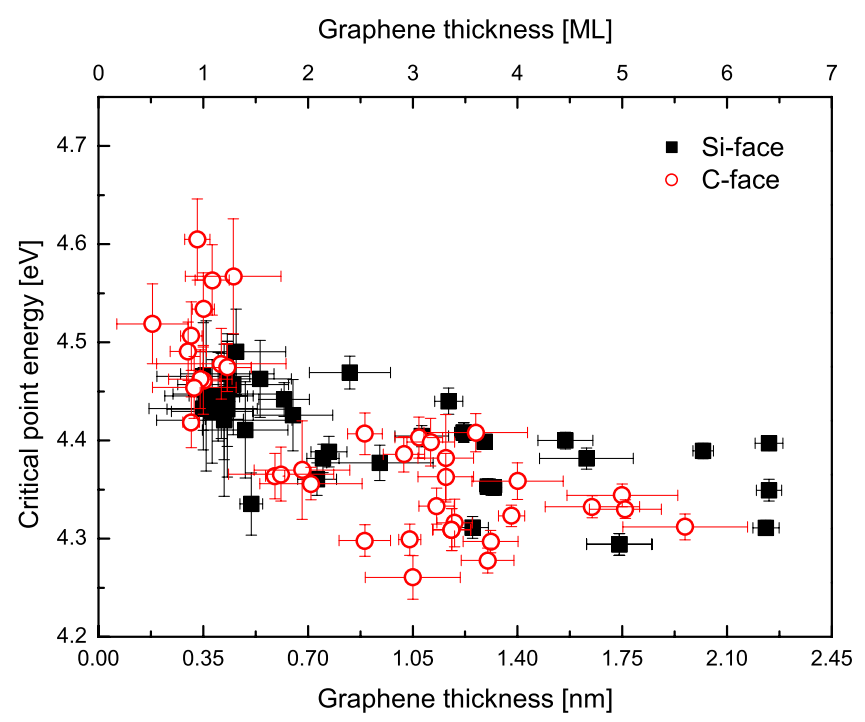

FIG. 3. Critical point energy versus epitaxial graphene thickness for the Siface (squares) and C-face (circles) of 3C-SiC(111).

is detected. This indicates a very different interface structure compared to the $\mathrm{Si}$-face $\mathrm{EG}$. Our recent studies indicate that the predominant type of defects on the $\mathrm{C}$-face of $3 \mathrm{C}-\mathrm{SiC}(111)$ is different from the twin boundaries found in Si-face 3C-SiC(111). On the C-face of 3C-SiC(111) small inclusions occur, each associated with $6 \mathrm{H}-\mathrm{SiC}$ formed around a screw dislocation. ${ }^{32}$ The distribution of these $6 \mathrm{H}-\mathrm{SiC}$ polytype defects is reminiscent of that of the small islands of high graphene coverage revealed in the interface layer map in Fig. 1(e).

Figs. 1(a), 1(c), 1(d), and 1(f) reveal a significant correlation between the graphene layer thickness and the freecharge-carrier scattering time. While areas of predominantly SLG reflect long scattering times, shorter scattering times correspond to the thick graphite-like islands. The reduction of scattering time indicates lower mobility across these areas and may be due to scattering across grain boundaries and/or scattering between the different graphene sheets.

Fig. 3 displays representative data points for the $\mathrm{CP}$ transition energy parameter and the graphene layer thickness obtained from the $\mu$-SE data analysis for both samples. While data uncertainty exists, the overall $\mathrm{CP}$ energy position is clearly and consistently blue-shifted with decreasing graphene thickness, a trend which was indicated by our recent SE study of EG on $3 \mathrm{C}-\mathrm{SiC}$ and $4 \mathrm{H}-\mathrm{SiC}$ using macroscopic spot size. ${ }^{21}$ In principle, the CP energy may depend on strain, doping, and number of layers. For free-standing SLG on quartz, the optical absorption is observed at $\sim 4.6 \mathrm{eV}$ while for graphite the absorption is red-shifted to $4.28 \mathrm{eV} .^{23}$ Therefore, the observed trend in Fig. 3 may be foremost related to the effect of number of graphene layers. In Fig. 3, the average CP energy for SLG on C-face is in very good agreement with the reported absorption energy of freestanding graphene $(\sim 4.6 \mathrm{eV})$, and which is presumably strain-free (or subjected to negligible strain). ${ }^{23}$ Thus, it is plausible to suggest that the interaction between graphene and the C-face 3C-Si(111) substrate is reduced and the EG does not experience significant strain. This is consistent with the LEED observation in Fig. 2(d) where no buffer layer is detected on the $\mathrm{C}$-face substrate. The $\mathrm{CP}$ energy for $\mathrm{C}$-face
SLG is systematically higher than the respective values for SLG on Si-face. This may be indicative of a more pronounced strain effect as a result of a stronger interaction of EG with the Si-face substrate. Indeed, we identified a buffer layer on the Si-face of the 3C-SiC(111) in Fig. 2(c), which in the case of Si-face $6 \mathrm{H}-\mathrm{SiC}$ and $4 \mathrm{H}-\mathrm{SiC}$ was shown to be strongly bonded to the substrate and be responsible for the strong interaction with EG.

In summary, large-area $\mu$-SE mapping and LEEM/ $\mu$-LEED investigations revealed critical correlations between surface reconstruction, graphene layer thickness, and electronic properties of EG grown on Si-face and C-face 3C-SiC. Growth of single monolayer graphene is demonstrated on both $\mathrm{Si}$ - and $\mathrm{C}$-face where large homogeneous domains with size up to $\sim 2 \times 2 \mathrm{~mm}^{2}$ are achieved on the Si-face. On the $\mathrm{C}$-face, the domains with homogeneous thickness are considerable smaller. The interface layer in this case also shows a distinctively different picture, with small uncorrelated nucleation sites that have high graphene coverage within the interface layer. We speculate that these sites may be associated with small pit defects on the C-face substrate that represent $6 \mathrm{H}-\mathrm{SiC}$ spiral growth inclusions. Furthermore, the maps of the free carrier scattering time show that the carrier mobility in the homogeneous areas of one monolayer EG is higher than the mobility in the thicker graphite islands. We find that the CP energy position associated with an exciton-enhanced van-Hove singularity in the density of states at $\sim 4.5 \mathrm{eV}$ blue shifts with decreasing the number of layers for both polarities. The analysis suggests that the interaction between EG and the substrate is stronger for the Si-face material. These results are consistent with our LEED observations indicating the formation of a $(6 \sqrt{3} \times 6 \sqrt{3}) \mathrm{R} 30^{\circ}$ buffer layer on the Siface and absence of any specific reconstruction on the $\mathrm{C}$-face samples.

We acknowledge support from the Swedish Research Council (VR) under Grant Nos. 2010-3848 and 2011-4447, the Swedish Governmental Agency for Innovation Systems (VINNOVA) under the VINNMER international qualification program, Grant No. 2011-03486 and the EU ConceptGraphene. We further acknowledge support by the National Science Foundation (MRSEC DMR-0820521, ECCS0846329, and EPS-1004094), the Department of Commerce NIST (70NANB11H165), the University of NebraskaLincoln, and the J. A. Woollam Foundation.

${ }^{1}$ C. Berger, Z. M. Song, T. B. Li, X. B. Li, A. Y. Ogbazghi, R. Feng, Z. T. Dai, A. N. Marchenkov, E. H. Conrad, P. N. First, and W. de Heer, J. Phys. Chem. B 108, 19912 (2004).

${ }^{2}$ C. Berger, Z. M. Song, X. B. Li, X. S. Wu, N. Brown, C. Naud, D. Mayou, T. B. Li, J. Hass, A. N. Marchenkov, E. H. Conrad, P. N. First, and W. A. de Heer, Science 312, 1191 (2006).

${ }^{3}$ C. Virojanadara, M. Syväjarvi, R. Yakimova, L. I. Johansson, A. A. Zakharov, and T. Balasubramanian, Phys. Rev. B 78, 245403 (2008).

${ }^{4}$ Y. M. Lin, H. Y. Chiu, K. A. Jenkins, D. B. Farmer, P. Avouris, and A. Valdes-Garcia, IEEE Electron. Device Lett. 31, 68 (2010).

${ }^{5}$ W. de Heer, C. Berger, M. Ruan, M. Sprinkle, X. Li, Y. Hu, B. Zhang, J. Hankinson, and E. Conrad, Proc. Natl. Acad. Sci. U.S.A. 108, 16900 (2011).

${ }^{6}$ Y. M. Lin, A. Valdes-Garcia, S. J. Han, D. B. Farmer, I. Meric, Y. N. Sun, Y. Q. Wu, C. Dimitrakopoulos, A. Grill, P. Avouris, and K. A. Jenkins, Science 332, 1294 (2011). 
${ }^{7}$ Y. Q. Wu, K. A. Jenkins, A. Valdes-Garcia, D. B. Farmer, Y. Zhu, A. A. Bol, C. Dimitrakopoulos, W. J. Zhu, F. M. Xia, P. Avouris, and Y. M. Lin, Nano Lett. 12, 3062 (2012).

${ }^{8}$ A. Ouerghi, M. Marangolo, R. Belkhou, S. El Moussaoui, M. G. Silly, M. Eddrief, L. Largeau, M. Portail, B. Fain, and F. Sirotti, Phys. Rev. B 82, 125445 (2010).

${ }^{9}$ A. Ouerghi, R. Belkhou, M. Marangolo, M. G. Silly, S. El Moussaoui, M. Eddrief, L. Largeau, M. Portail, and F. Sirotti, Appl. Phys. Lett. 97, 161905 (2010).

${ }^{10}$ J. L. Tadesco, G. G. Jernigan, J. C. Culberston, J. K. Hite, Y. Yang, K. M. Daniels, R. L. Meyers-Ward, C. R. Eddy, Jr., J. A. Robinson, K. A. Trumbull, M. T. Wetherington, P. M. Campbell, and D. K. Gaskill, Appl. Phys. Lett. 96, 222103 (2010).

${ }^{11}$ X. Weng, J. A. Robinson, K. Trumbull, R. Cavalero, M. A. Fanton, and D. Snyder, Appl. Phys. Lett. 100, 031904 (2012).

${ }^{12}$ L. I. Johansson, S. Watcharinyanon, A. A. Zakharov, T. Iakimov, R. Yakimova, and C. Virojanadara, Phys. Rev. B 84, 125405 (2011).

${ }^{13}$ M. Schubert, Infrared Ellipsometry on Semiconductor Layer Structures: Phonons, Plasmons and Polaritons (Springer, New York, 2004), Vol. 209.

${ }^{14}$ H. Fujiwara, Spectroscopic Ellipsometry: Principles and Applications (William Andrew Publishing, 2007).

${ }^{15}$ V. Darakchieva, T. Hofmann, M. Schubert, B. E. Sernelius, B. Monemar, P. O. A. Persson, F. Giuliani, E. Alves, H. Lu, and W. J. Schaff, Appl. Phys. Lett. 94, 022109 (2009).

${ }^{16}$ V. Darakchieva, M. Schubert, T. Hofmann, B. Monemar, C. L. Hsiao, T. W. Liu, L. C. Chen, W. J. Schaff, Y. Takagi, and Y. Nanishi, Appl. Phys. Lett. 95, 202103 (2009).

${ }^{17}$ F. J. Nelson, V. K. Kamineni, T. Zhang, E. S. Comfort, J. U. Lee, and A. C. Diebold, Appl. Phys. Lett. 97, 253110 (2010).

${ }^{18}$ I. Santoso, P. K. Gogoi, H. B. Su, H. Huang, Y. Lu, D. Qi, W. Chen, M. A. Majidi, Y. P. Feng, A. T. S. Wee, K. P. Loh, T. Venkatesan, R. P. Saichu, A. Goos, A. Kotlov, M. Rübhausen, and A. Rusydi, Phys. Rev. B 84, 081403 (2011).
${ }^{19}$ W. Strupinski, K. Grodecki, A. Wysmolek, R. Stepniewski, T. Szkopek, P. E. Gaskell, A. Gruneis, D. Haberer, R. Bozek, J. Krupka, and J. M. Baranowski, Nano Lett. 11, 1786 (2011).

${ }^{20}$ M. Zhou, F. L. Pasquale, P. A. Dowben, A. Boosalis, M. Schubert, V. Darakchieva, R. Yakimova, L. Kong, and J. A. Kelber, J. Phys.: Condens. Matter 24, 072201 (2012).

${ }^{21}$ A. Boosalis, T. Hofmann, V. Darakchieva, R. Yakimova, and M. Schubert, Appl. Phys. Lett. 101, 011912 (2012).

${ }^{22}$ J. M. Weber, V. E. Calado, and M. C. M. van de Sanden, Appl. Phys. Lett. 97, 091904 (2010).

${ }^{23}$ V. G. Kravets, A. N. Grigorenko, R. R. Nair, P. Blake, S. Anissimova, K. S. Novoselov, and A. K. Geim, Phys. Rev. B 81, 155413 (2010).

${ }^{24}$ M. Losurdo, M. M. Giangregorio, and G. Bruno, J. Phys. Chem. C 115, 21804 (2011).

${ }^{25}$ U. Wurstbauer, C. Röling, U. Wurstbauer, W. Wegscheider, M. Vaupel, P. Thiesen, and D. Weiss, Appl. Phys. Lett. 97, 231901 (2010).

${ }^{26}$ O. Albrektsen, R. L. Eriksen, S. M. Novikov, D. Schall, M. Karl, S. I. Bozhevolnyi, and A. C. Simonsen, J. Appl. Phys. 111, 064305 (2012).

${ }^{27}$ A. Matković, A. Beltaos, M. Milićević, U. Ralević, D. J. B. Vasić, and R. Gajic, J. Appl. Phys. 112, 123523 (2012).

${ }^{28}$ T. Hofmann, A. Boosalis, P. Kühne, C. M. Herzinger, J. A. Woollam, D. K. Gaskill, J. L. Tadesco, and M. Schubert, Appl. Phys. Lett. 98, 041906 (2011).

${ }^{29}$ R. Vasiliauskas, M. Marinova, M. Syväjärvi, R. Liljedahl, G. Zoulis, J. Lorenzzi, G. Ferro, S. Juillaguet, J. Camassel, E. K. Polychroniadis, and R. Yakimova, J. Cryst. Growth 324, 7 (2011).

${ }^{30}$ H. Hibino, H. Kageshima, F. Maeda, M. Nagase, Y. Kobayashi, and H. Yamaguchi, Phys. Rev. B 77, 075413 (2008).

${ }^{31}$ N. Camara, G. Ruis, J. R. Huntzinger, A. Tiberj, L. Magaud, N. Mestres, P. Godignon, and J. Camassel, Appl. Phys. Lett. 93, 263102 (2008).

${ }^{32}$ R. Vasiliauskas, S. Juillaguet, M. Syväjävi, and R. Yakimova, J. Cryst. Growth 348, 91 (2012). 